\title{
Research and Design on the on-line Monitoring for Rainwater Leakage of Floors on Substation Roofs
}

\author{
Xin Tang ${ }^{1}$, Gang Chen ${ }^{2}$, Bin $\mathrm{Hu}^{3}$, jinbo Zhang ${ }^{4}$, Bin $\mathrm{Chen}^{5}$ and Shuai $\mathrm{Lu}^{6}$ \\ 1,2,3 The Jiaxing state grid, Jiaxing, Zhejiang ,China \\ ${ }^{4,5,6}$ Hohai University College of Internet of Things Engineering, Changzhou, Jiangsu, China
}

\begin{abstract}
In order to reduce the safety loophole for substation caused by rainwater leakage of roof, this paper proposed an on-line monitoring for rainwater leakage of substation roof based on a parallel plate capacitor sensor. This method is based on the capacitance sensor in the composition of installed metal plate between slabs, when there is rainwater leakage in the floor, medium of capacitance will change obviously, and it can judge that whether the floor rainwater leakage occurs according to the characteristics. With the above principle, this paper designed an on-line monitoring device for rainwater leakage of substation roof. The practical test showed that the device has the advantages of usableness, low cost and accurate judgement. It can satisfy the requirement of the substation roof rainwater leakage monitoring.
\end{abstract}

Keywords-substation; roof; rainwater leakage; plate condenser; on-line monitoring

\section{INTRODUCTION}

As a kind of industrial buildings, substation construction has its generality in the aspect of waterproof impermeability. And for indoor substation construction, due to the special requirements of the electrical equipment, the water seepage of important electrical equipment room might influence the electrical equipment safety. Recently, with the high rapid development of electric power construction, a large number of indoor substation buildings have come into use. From the point of the usage of the substation, there is a lot of leakage phenomenon which produces effects and hidden danger on the nattiness, beauty and safety of the substation buildings. As for traditional method of building leakage monitoring, its instrument operation is relatively complex, its requirement for monitoring personnel technical level is higher, and its cost is rather high, so it cannot make a wide range of online monitoring. Accordingly, this paper put forward a new method of on-line monitoring for roof rainwater leakage of substation floor, and the method adopted the principle of capacitance sensor which was based on the capacitance sensor in the composition of installed metal plate between slabs, when there is water leakage on the floors, the medium of capacitance will change obviously. It can judge whether water leakage of floor occurs according to the above characters, and it makes use of the designed acquisition circuit to measure the value of capacitance. Through the conversation of capacitance value to frequency signal by conditioning circuit, and making real-time display for corresponding frequency values according to leakage level on the water leakage monitoring device, the device can improve the real-time and reliability of on-line monitoring for roof rainwater leakage of transformer substation, and can reduce maintenance costs, at the same time, it can reduce the hidden danger of electrical equipment safety. ${ }^{[1-9]}$

\section{A. The Principle of Parallel Plate Capacitance sensor}

The schematic diagram of parallel plate capacitance sensor is shown in Figure I . It is made up of two pieces of mental which are keeping close, mutual parallel and having the same size. Assuming that the area and the inner surface distance of each pole plate is $\mathrm{s}$ and $\mathrm{d}$ respectively and the plate dimension is far above the inner surface distance. The capacity of positive electricity of a plate is $+q$ and the capacity of negative electricity of $B$ plate is -q. The charge can be regarded evenly distributing on the plate except the edges when considering the area of each pole plate is huge, and their inner surface distance is small. When edge effect is ignored, the capacitance is:

$$
C=\varepsilon \frac{S}{d}
$$

In the equation (1), is the dielectric constant material in the sensor.

Assuming that the gravity of housing impervious layer (without proof) is $\alpha$, the gravity of water in the housing impervious layer is $\beta$, assuming the weight of the sensor in water house impervious layer is $\mathrm{G}$, and there are:

$$
\begin{aligned}
& G=G_{1}+G_{2} \\
& G_{1}=\alpha S_{1} d \\
& G_{2}=\beta S_{2} d
\end{aligned}
$$

In the equation (2), G1 is the weight of the impervious layer for the house, and G2 is the weight of water in the housing impervious layer.

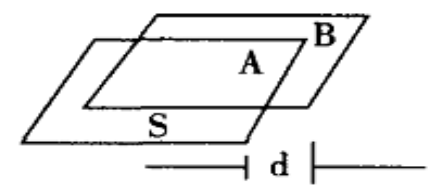

FIGURE I. THE PRINCIPLE OF PARALLEL PLATE CAPACITOR 
Assuming that $S_{1}$ and $S_{2}$ are the equivalent area of housing impervious layer and water, and the corresponding equivalent capacitance are respectively $C_{1}$ and $C_{2}$, then there are:

$$
\begin{aligned}
& C=C_{1}+C_{2} \\
& S=S_{1}+S_{2}
\end{aligned}
$$

From the equation (1)、(2)and(3), the capacitance $C_{x}$ of sensors which installed housing impervious layer with water is:

$$
C x=\frac{S}{d}\left(\frac{S_{1}}{S} \varepsilon_{1}+\frac{S_{2}}{S} \varepsilon_{2}\right)
$$

In the equation (5), $\varepsilon_{1}$ and $\varepsilon_{2}$ are respectively dielectric constant of housing impervious layer and water. The moisture content in the housing impervious layer $\delta$ is defined as:

$$
\delta=\frac{G_{2}}{G_{1}+G_{2}} 100 \%
$$

Then we can get from(4)、(5)、(6)and $\beta \approx 1$ that:

$$
C x=\frac{S}{d} \varepsilon_{1}+\frac{S}{d}\left(\varepsilon_{2}-\varepsilon_{1}\right) \frac{\delta}{(1-\delta) / \alpha+\delta}
$$

It can be known from (7), the first item is the capacitance of sensors with dry housing impervious layer which is expressed as $C_{O}$, and the second is the changed capacitance caused by the water in the housing impervious layer whose variation is expressed as $\Delta C x$. Then there is:

$$
\begin{gathered}
C x=C o+\Delta C x \\
C o=\frac{S}{d} \varepsilon_{1} \\
\Delta C x=\left(\varepsilon_{2}-\varepsilon_{1}\right) \frac{S}{d} \frac{\delta}{(1-\delta) / \alpha+\delta}
\end{gathered}
$$

The equation (10) shows that $\Delta C x$ is related to moisture content $\delta$.For moisture content and sensors ${ }^{[3-5]}$, it can judge the value of $\delta$ by the measurement of $\Delta C x$, that is the principle of capacitance transducer used for housing impervious layer moisture content measurement. Finally, it gets the voltage between the plates by the change of capacitance. ${ }^{[10]}$
II. The Hardware Circuit Design Of Parallel Plate CAPACITOR FOR FLOOR ON THE ROOF RAINWATER LEAKAGE MONITORING DEVICE

A. The Overall Structure Design of Parallel Plate Capacitor for Floor on the Roof Rainwater Leakage Monitoring Device

For the old floors, in order to prevent destruction to waterproof layer, it needs to install thin metal plates on the upper and lower layer to constitute a parallel plate capacitor sensor, then the waterproof layer is the capacitor dielectric between the plates. For the floors of new buildings, it uses capacitance plate as the single capacitance plate which includes sheet metal, rubber plate, connecting wires. One end of wires are connected to thin copper foil, the other end are connected to the input of signal pretreatment circuit, and the thin metal is poured inside the rubber sheet. When measuring, the single capacitance is placed on the roof which makes up a capacitance sensor with reinforcing roils laying on the house, and the waterproof is the dielectric between the plates of single capacitor. The on-line rainwater leakage monitoring device for substation roof is mainly based on the basis of obvious change of dielectric when there is rainwater leakage in the floor, then we can estimate whether rainwater leakage occurs. Through the real-time collection of the value of the capacitance sensor, combined with the same day and meteorological conditions in recent days, the on-line monitoring device can make comprehensive judgement about rainwater leakage of floor on the roof. The device includes the parallel plate capacitor sensor module, the signal conversion module, the microprocessor, the storage module, the rainfall measuring module, the display module, the keys module, the RS485 communication module, the power supply module, etc. The basic structure block diagram is shown in FIGURE II .

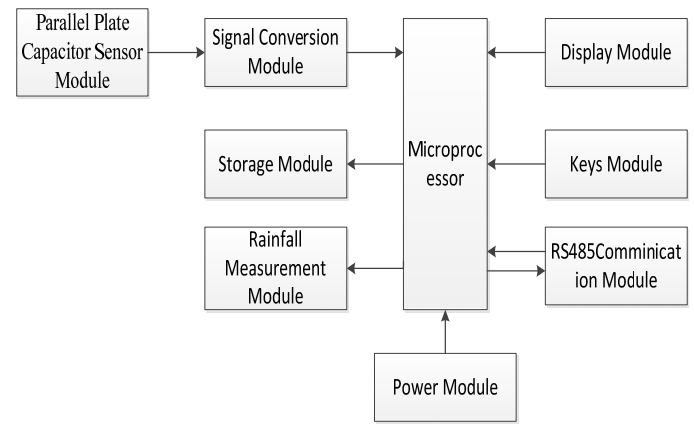

FIGURE II. THE PRINCIPLE STRUCTURE OF ON-LINE MONITORING DEVICE FOR RAINWATER LEAKAGE

\section{B. The Measurement of Capacitance sensor Value for Monitoring Rainwater Leakage in the Roof}

The capacitance sensor for monitoring rainwater leakage in the roof is mainly based on the obvious change of capacitor dielectric when there is rainwater leakage, thus judging whether rainwater leakage occurs. This paper adopts the oscillation circuit with NE555, which converts the change of capacitance value to frequency signal, and the frequency of oscillation circuit is associated with resistance and capacitance. When the resistance is fixed and the capacitance is variable, 
the capacitance value can be calculated by measuring the frequency.

\section{The Software Design Of Parallel Plate CAPACITOR SENSOR FOR FLOORS ON THE ROOF RAINWATER LEAKAgE MONITORING DEVICE}

The software flow diagram of parallel plate capacitor sensor for floors on the roof rainwater leakage monitoring device is shown in Figure III. It converts the capacitance value obtaining from the parallel plate capacitance sensor to frequency signal and inputs to the measurement chip. The on-line monitoring device for floors on the roof can make real-time display the leakage frequency of the current measured substation roof, and the value of frequency is corresponding to the degree of building leakage, the smaller the frequency values is, the more serious the housing leakage will be. When the frequency is lower than the setting alarm value [6-10], the leakage detection device will alarm immediately to notify the management. The alarm value can set by keypress according the actual demand.

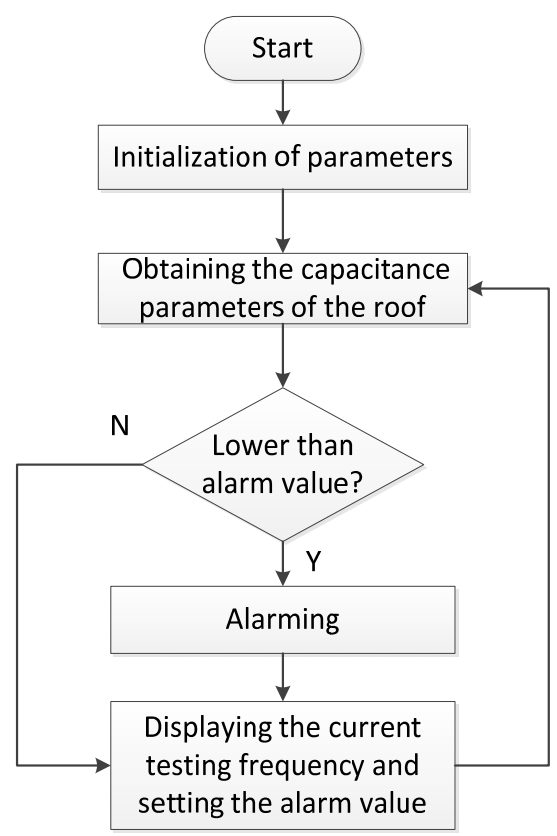

FIGURE III. THE SOFTWARE FLOW CHART OF RAINWATER LEAKAGE MONITORING SYSTEM

When monitoring the floor on the roof, this device evenly divided the roof into a number of monitor units, and the detector collects and stores inspection data of each unit, then it maps the roof scanning data which can get whole roof leakage distribution, thus judging the location and size of wet source. According to the detection of standard test specimen, it demarcated the corresponding relation between monitoring frequency and moisture rate of impermeable layer. The monitoring data is divided into four gears, and the moisture content is $\mathrm{A}>\mathrm{B}>\mathrm{C}>\mathrm{D}$ from high to low in turn, then drawing isohume, we can get water leakage location of the roof according to the isohume.

\section{CONCLUSION}

Leak detection is one of the common concerned problems in the field of building quality detection and maintenance. In recent years, a large number of testing equipment at home and abroad tend to be more portable, automatic and intelligent but the rainwater leakage on-line monitoring of the floor on the roof is in the development phase at home. This paper presents a new rainwater leakage on-line monitoring method for the floor on the roof of substation. In addition, the development of on-line monitoring device has the functions of automatic monitoring, automatic recording, and automatic analysis etc. The application of this device can greatly improve the real-time performance and reliability of rainwater leakage on-line monitoring of substation and reduce the hidden danger brought by the rainwater leakage of substation.

\section{REFERENCES}

[1] Meng Yanhua, Li Yun, The research on flat roof leakage detection data acquisition system [J]. Shanxi building, 2006, 32(19):331-332

[2] Xue Shumin, Wang Wenhao, Xia Lu. The application of infrared thermal image technology in the housing leakage detection[J]. The waterproof of Chinese buildings, 2012,22:38-39

[3] Yang Jianjun, Chen Xing. The design of the WSJY- type 2 roof leakage detector [J]. The modern electronic technology, 2011,34(22):132-133

[4] Xia Zhongxian. The detection and analysis discussion of housing cracks and leakage [J]. Residential science and technology, 2011,S1:273-274

[5] Li Yun. The research on capacitance sensor leakage detection method [J]. Henan technology, 2013,23:55-56

[6] Pan Junfeng, Neng Changxin, Zhang Yun, etc. The application of portable leakage detection device in landfill impermeable layer integrity testing [J]. Research of Environmental Sciences, 2008,21(6):43-46

[7] Zhu Sihan,Wang Bin, Wang Jing,etc. The development and research on solid waste Landfill leakage detection system software [J]. Microcomputer \& Its Applications 2014, 33(8):7-9

[8] Yingchieh Ho,Katherine Shu-Min Li, Sying-Jyan Wang. Leakage Monitoring Technique in Near-threshold Systems with a Time-based Bootstrapped Ring Oscillator[J]. Asian Test Symposium,2013,22:91-96

[9] Ludwig Hoegner, Uwe Stilla. Thermal leakage detection on building facades using infrared textures generated by mobile mapping[J]. Urban Remote Sensing Joint Event,2009

[10] Zhang Jinbo, Wang Jun, Fan Meirong. The design of moisture content sensor for gain determination [J]. Micro computer information, 2005,21(9):144-145 